(C)2008 IEEE. Personal use of this material is permitted. However, permission to reprint/republish this material for advertising or promotional purposes or for creating new collective works for resale or redistribution to servers or lists, or to reuse any copyrighted component of this work in other works must be obtained from the IEEE. 


\title{
Design and Study of a Switch Reactor for Central Queensland SWER system
}

\author{
M. R. Hesamzadeh, N. Hosseinzadeh, and P. J. Wolfs, CQ University, Australia
}

Abstract-- Single Wire Earth Return (SWER) systems are a widely applied, low cost electrification method used in many rural areas. In Central Queensland a single SWER system supplying approximately $100 \mathrm{~kW}$ may extend more than $300 \mathrm{~km}$. Many SWER systems include shunt reactors to control the effects of the line charging capacitance. One effect, the Ferranti effect, causes the line voltage to rise with the distance. In three phase distribution systems this effect is not visible but in SWER systems, this effect makes it difficult to maintain the consumers supply within the acceptable regulation range. As the second effect, the loading of the SWER system supply transformer increases. Controllable shunt reactors are used as one solution to the aforementioned problems.

Stanage Bay feeder in Central Queensland area has been chosen for the installation of the designed shunt reactor. Stange Bay feeder is supplied by an isolating transformer with the total capacity of $150 \mathrm{kVA}$ and the voltage level of $22 \mathrm{kV}$.

Using the Stanage Bay feeder, this paper details the process of design and simulation of a suitable switch reactor. This step has been carried out by firstly, the design of the switch reactor and secondly, the proper modelling of the designed reactor for the voltage regulation studies.

Index Terms-- SWER system, Rural electrification, Switch reactor, Central Queensland

\section{INTRODUCTION}

$\mathrm{S}_{\mathrm{i}}^{\mathrm{i}}$ ingle Wire Earth Return systems have been widely installed in Australia and New Zealand over 50 years, [13]. This approach is promoted by the World Bank as a lowest cost technology and will find growing applications in bringing supply to the estimated two billion persons globally without power, [3]. SWER systems typically supply loads of $100 \mathrm{~kW}$ to $200 \mathrm{~kW}$ scattered over a line length that might exceed $300 \mathrm{~km}$.

In the state of Queensland, a SWER task force has been established to investigate the load growth issues faced by these systems. An important option is to apply new technologies into aging SWER systems to release capacity for load growth.
Many long SWER systems include shunt reactors to control the effects of the line charging capacitance. In SWER systems, as a result of Ferranti effect, it is very difficult to maintain the consumer voltage within the acceptable regulation range. The line charging current without reactors may be as high as twice the SWER system isolating transformer rating.

The industry has always recognized the immediate advantages in removing the reactors at higher loads. Switchable reactors at $19.05 \mathrm{kV}$ will require a high voltage motorized switch, a voltage transformer, and a designed control element. The costs of switch and voltage transformer are a bottleneck in final cost evaluation of the technology.

Alternatively, it is possible to install the switch reactor at lower voltages on a transformer secondary. $25 \mathrm{kVA}$ consumer transformers are produced in large quantities and are moderately priced. In this case, the $19.05 \mathrm{kV}$ switch reactors could be replaced by $480 \mathrm{~V}$ inductors connected across the centre tap transformer of 240-0-240V. Thyristor controlled reactors, contactor switched reactors, and contactor controlled reactors connected via dedicated consumer transformers are three proposed solution for the SWER system voltage problem.

This paper explains the design and study process of a typical switch reactor for one of the SWER systems in the state of Queensland, Australia. The paper is organized in four sections. Introduction of the paper is explained in section I. Problem formulation and the modelling of the SWER system is explained in section II. Section II is dedicated to the switch reactor design and associated studies. And finally conclusion in section IV would close the paper.

\section{PRoblem Formulation AND MODELLING OF THE SWER SYSTEM}

Stanage Bay SWER system is one of the many SWER branches supplied by the $66 / 22 \mathrm{kV}$ Pandoin Zone substation. It is connected through a $22 \mathrm{kV}(3 \mathrm{ph}) / 19.05 \mathrm{kV}(1 \mathrm{ph})$ and $150 \mathrm{kVA}$ isolating transformer. The connection point of the isolating transformer is $70 \mathrm{~km}$ away from the Pandoin Zone substation. The connection schematic of the isolating transformer is shown on figure 1 . 


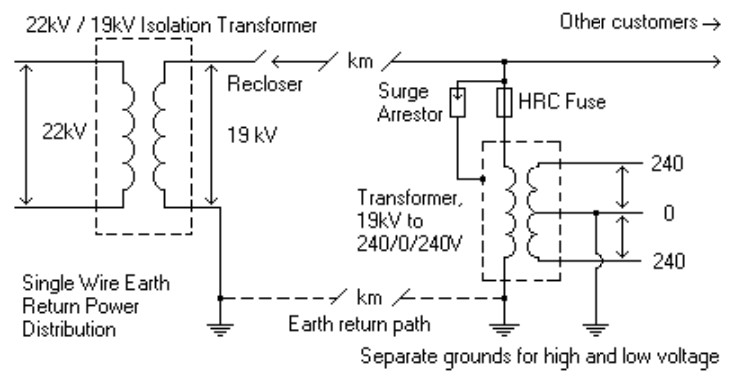

Figure 1, the connection schematic of the isolating transformer

Table 1 represents the electrical specifications of isolating transformer used for Stanage Bay feeder.

TABLE 1: ELECTRICAL CHARACTERISTICS OF ISOLATING TRANSFORMER OF STANAGE BAY FEEDER

\begin{tabular}{|c|c|c|c|c|c|c|}
\hline $\begin{array}{c}\text { Un1 } \\
(\mathrm{kV})\end{array}$ & $\begin{array}{c}\text { Un2 } \\
(\mathrm{kV})\end{array}$ & $\begin{array}{c}\text { Smax } \\
(\mathrm{kVA})\end{array}$ & uk (\%) & ur (\%) & $\begin{array}{c}\text { Vector } \\
\text { Group }\end{array}$ & $\begin{array}{c}\text { R0/X0 } \\
(\text { p.u. })\end{array}$ \\
\hline 22 & 33 & 150 & 4.1231 & 1.6 & YND1 & 0.4211 \\
\hline
\end{tabular}

A typical load pattern of the isolating transformer recorded during the January 2007 with 5 minutes intervals has been shown through figure 2.

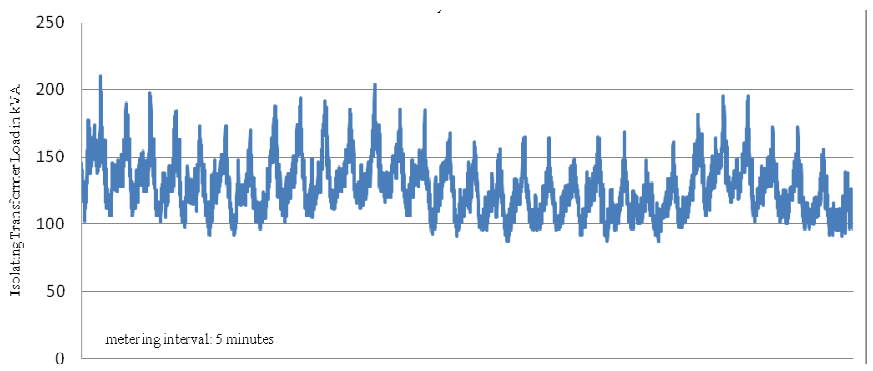

Figure 2, the load pattern of the isolating transformer recorded during the January 2007

$19.05 \mathrm{kV}$ Stanage Bay SWER system has the total length of around $95 \mathrm{~km}$ and total maximum connected demand of $180 \mathrm{kVA}$ on average. The conductor is $3 / 4 / 2.5 \mathrm{ACSR} / \mathrm{GZ}$ with the table 2 parameters.

TABLE 2: CONDUCTOR SPECIFICATIONS OF THE STANAGE BAY FEEDER SYSTEM

\begin{tabular}{|c|c|}
\hline Conductor & Parameters \\
\hline $3 / 4 / 2.5$ & R0: $2.02 \Omega / \mathrm{km} ; \mathrm{X} 0: 0.802 \Omega / \mathrm{km} ; \mathrm{B} 1: 2.086 \mu \mathrm{mho} / \mathrm{km}$ \\
$\mathrm{ACSR} / \mathrm{GZ}$ & \\
\hline
\end{tabular}

The Stanage Bay SWER system conductors are modelled as the Pi for the system studies.

Since the LV consumers supplied by the Stanage Bay SWER system have the same pattern of consumption, a recorder has been installed close to the installation point of the switch reactor and the collected data has been used for the loading of the other consumer transformers. The pattern of consumption for all consumer transformers is like the reference consumer transformer, to which the data logger is connected. The scaling factor calculated based on the maximum capacity of a specific transformer and the reference transformer is used for finding the load of the consumer transformer.

The following two formulas are used for the load allocation to the consumer transformers based on the reference consumer transformer recorded data.

$$
\begin{aligned}
& S F_{n}=\frac{S_{\operatorname{Max}(n)}}{S_{\operatorname{Max}(r e f)}} \\
& S_{n}(k V A)=S F_{n} \times S_{r e f}(k V A)
\end{aligned}
$$

Figure 3 shows the real loading data of the reference consumer transformer on the second of January and the scaling factor concept.

The geographical distribution of the Stanage Bay SWER system and the modelled network in PSS-SINCAL are shown through figures 4 and 5 .

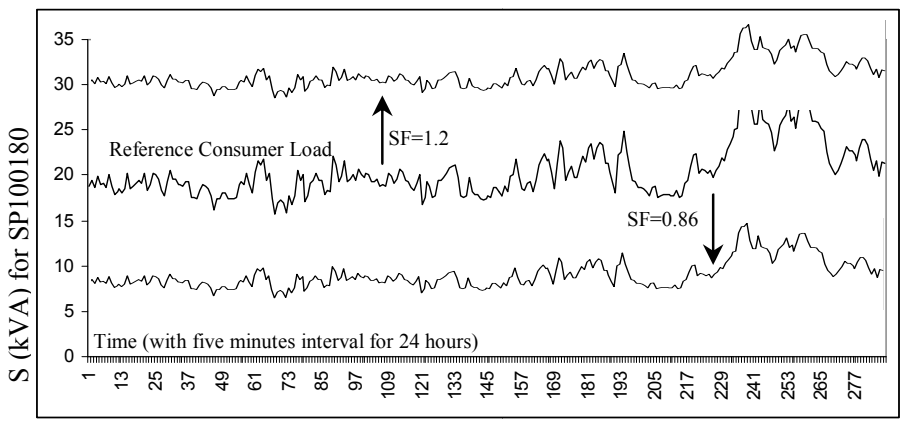

Figure 3, loading data of the reference consumer transformer on the second of January

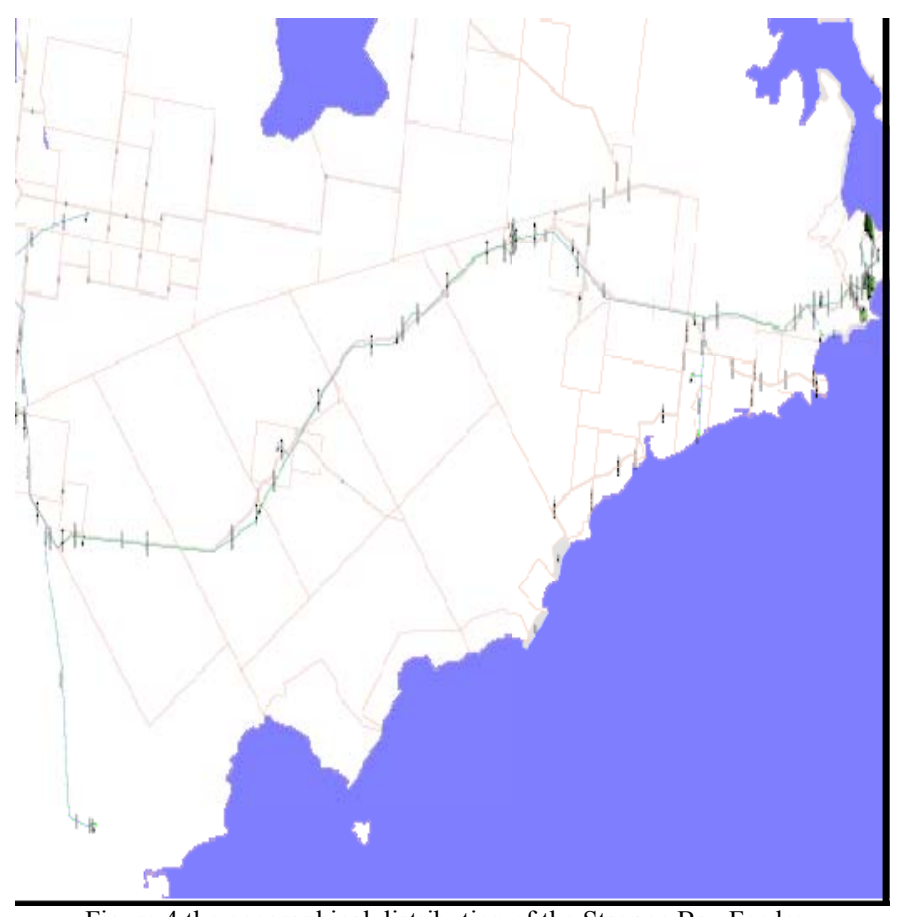

Figure 4 the geographical distribution of the Stanage Bay Feeder 


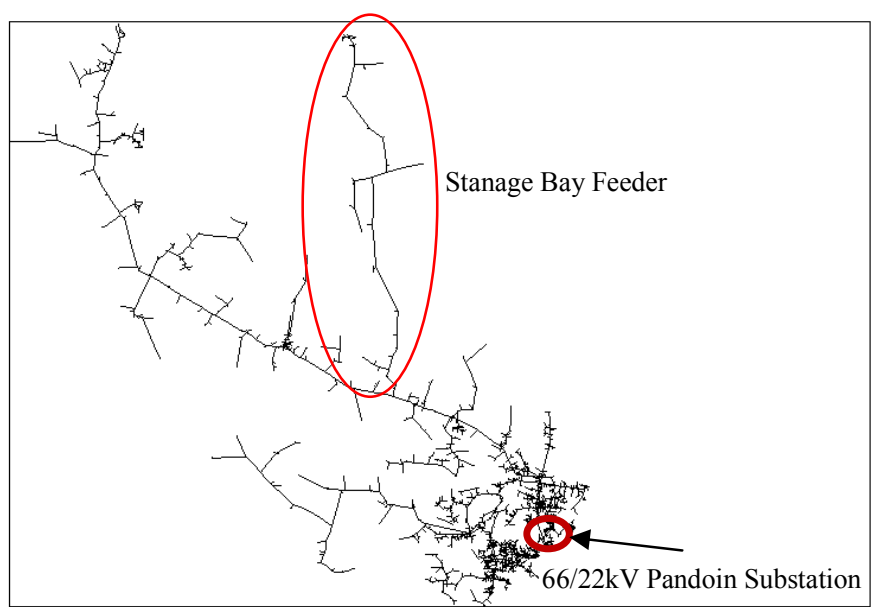

Figure 5 the 66/22kV Pandoin substation and Stanage Bay Feeder modelled in PSS-SINCAL

Considering the aforementioned specifications of the under study SWER system, the next section would cover firstly, the switch reactor design and then studying the Stanage Bay feeder with and without switch reactor for validating the effectiveness of the scheme.

\section{SWITCH REACTOR DESIGN AND ASSOCIATED STUDIES}

This section firstly explains the design process of the switch reactor and then studies the effects of installed switch reactor in two scenarios. First scenario models the Stanage Bay feeder without switch reactor and the second scenario models the Stanage Bay with switch reactor.

\section{A. Design of the switch reactor}

The design of the controlled reactor system can be seen in two stages. The first stage is the reactor design and the second one is the controller design. These two stages are explained through subsections A.1 and A.2.

\section{A.1 Reactor Design}

Two $460 \mathrm{~V}, 54 \mathrm{mH}, 12.5 \mathrm{kVA}$ reactors are used. The reactors were bench tested for temperature rise and quality factor. Figure 6 shows a $12.5 \mathrm{kVA}$ reactor in and enclosure with parallel capacitors and current transducers. The inductors were tested in a parallel resonant arrangement to allow easy excitation with a small variac.

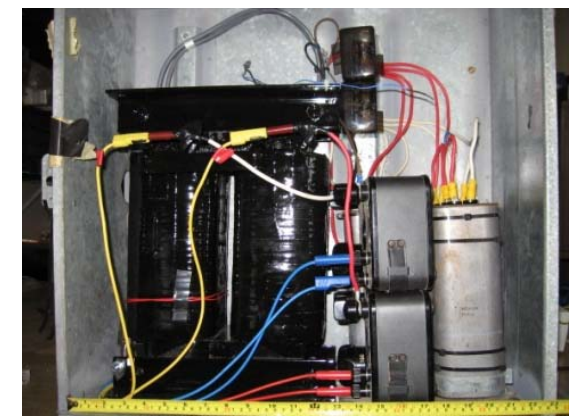

Figure 6 bench testing of reactor quality factor and temperature rise
On the left limb of the inductor a red search coil can be seen. The search turn voltage can be integrated by a digital oscilloscope with a mathematical capacity to produce an indication of flux and flux density B. In combination with the inductor current, a direct indicator of magnetomotive force, $\mathrm{H}$, a B-H curve can be developed and the susceptibility of the inductor to saturation can be determined. The inductors showed no saturation at $532 \mathrm{~V}$, a $16 \%$ overvoltage and the limit of the test system. This corresponds to a reactive power of $17 \mathrm{kVA}$. Magnetically the design is conservative.

The measured inductor $\mathrm{Q}$ is 55. A thermocouple was inserted between the coil former and core to estimate the hot spot temperatures. This thermocouple could be inserted more than $50 \mathrm{~mm}$ and is a good indicator of internal temperature. The core was seen to stabilize in an eight-hour temperature rise test. This resulted in a $35^{\circ} \mathrm{C}$ surface temperature rise and a $50^{\circ} \mathrm{C}$ rise at the coil/core interface. The air temperature was $44^{0} \mathrm{C}$ and the maximum observed temperature at the coil core interface was $94^{\circ} \mathrm{C}$. The inductor insulation system is class $\mathrm{H}$ $\left(180^{\circ} \mathrm{C}\right)$. The insulation life is estimated at better than 20 years.

A 25kVA SWER transformer has been used for testing. It was tested based on the open circuit and short circuit tests which could be conducted by energising the transformer from the low voltage side. The short circuit impedance, $3.3 \%$ was in line with the values used for the simulation models and in agreement with the name plate data. The magnetising current, $0.36 \%$, and core loss, $0.21 \%$, was significantly smaller than expected for the transformers which are connected to the system.

The transformer has two $250 \mathrm{~V}$ windings. This allowed further testing to be performed on the effects of DC unbalance which could be present for a thyristor controlled reactor design. Figure 7 shows an arrangement in which the transformer could be easily subjected to a DC offset current.

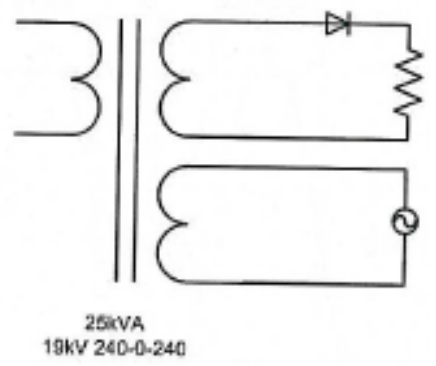

Figure 7 DC offsets on core magnetisation

In designing the controller, a decision was made to follow a low technology analogue design path to produce a controller that was robust, easy to test, and ultimately very easy to maintain. The controller contains the following functional blocks;

- A linear power supply that is connected from the line to neutral; 
- A precision differential amplifier;

- A precision rectifier;

- An extreme low pass second order filter;

- An error amplifier;

- Two hysteresis comparators;

- Relay drivers;

- Relay with potential contacts.

The controller directly drives the $240 \mathrm{~V}$ holding coils of two 63A three phase contactors. Each contactor drives one switched inductor. The switch reactor unit requires a three wire $240 \mathrm{~V}-0-240 \mathrm{~V}$ connection. While the reactors are switched line to line the control unit power supply and holding coils are $240 \mathrm{~V}$ devices and are powered from one line to neutral.

Based on the design of the switch reactor, three scenarios of load have been studied. In the heavy-load scenario, all two inductors are out. Reactor number 1 with the capacity of $12.5 \mathrm{kVA}$ comes in during the medium -load scenario and finally, both reactors are in during the light-load scenarios.

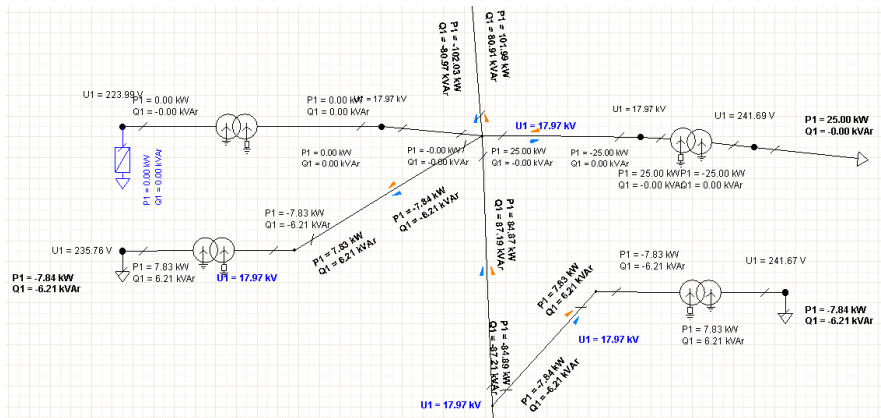

Figure 8: The heavy-load scenario when both reactors are out

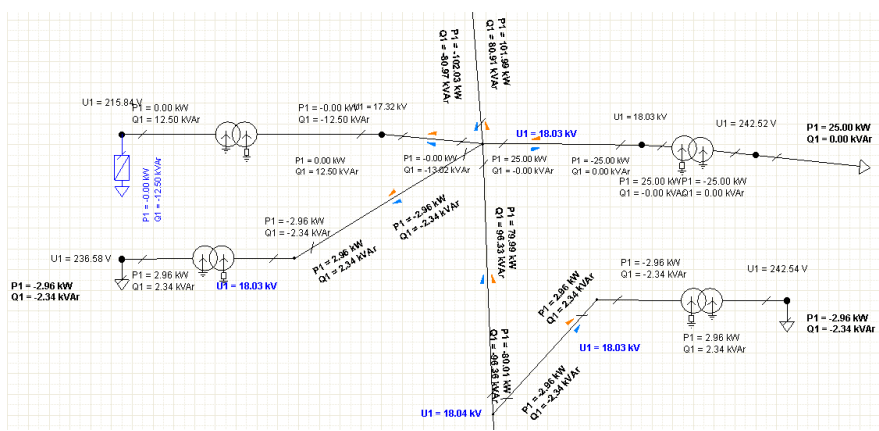

Figure 9: The medium-load scenario when only one reactor with capacity of $12.5 \mathrm{kVA}$ is in

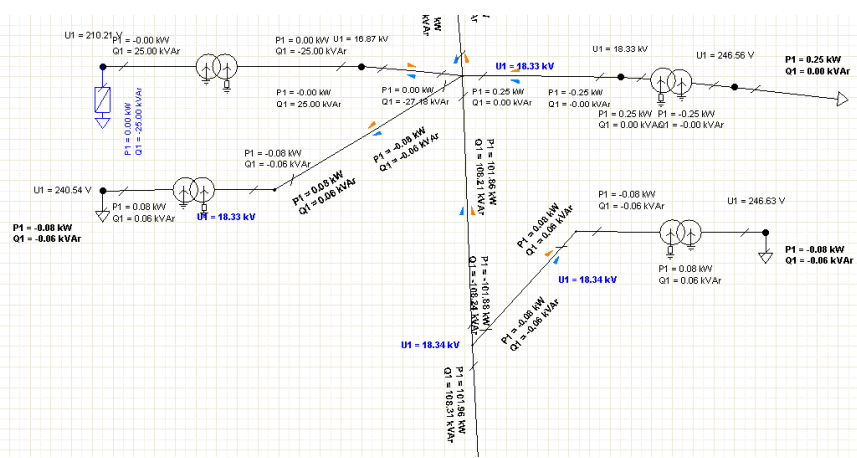

Figure 10: The light-load scenario when both reactors are in with the total capacity of $25 \mathrm{kVA}$

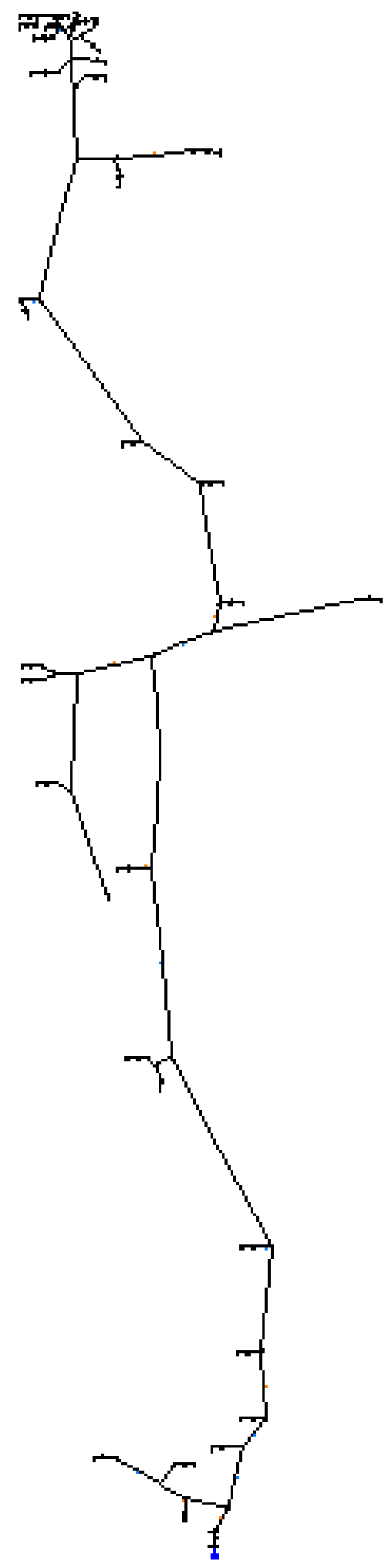

Figure 11: Single line diagram of the Stanage Bay SWER system

As it is clear from figure 8 through 10 , in heavy load scenario both reactor are out and the voltage at the connection point of the switch reactor is about $17.97 \mathrm{kV}$ line-to-neutral. In case of medium load scenario only one of the inductors is in and the voltage at the connection point of the switch reactor is about $18.03 \mathrm{kV}$. Finally, for light-load scenario when both reactors 
are in with the total capacity of $25 \mathrm{kVA}$, the voltage at the connection point of the designed switch reactor is around $18.33 \mathrm{kV}$.

Based on the field data, figure 12, shows the switching pattern of the reactors for one operating day. Figure 13 is the voltage pattern of the connection point of the switch reactor. Figures 12 and 13 are based on the practical data collected from the installation point of the switch reactor.

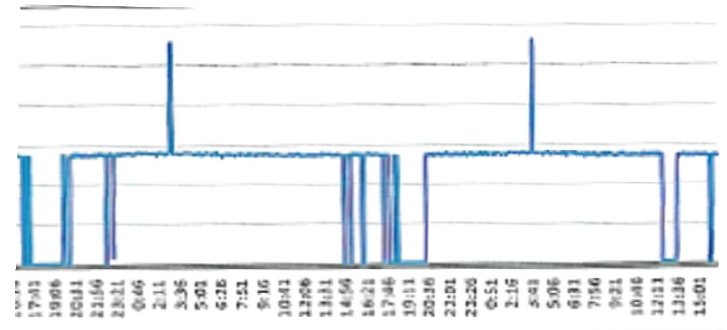

Figure 12: switching pattern of the switch reactor for one operating day

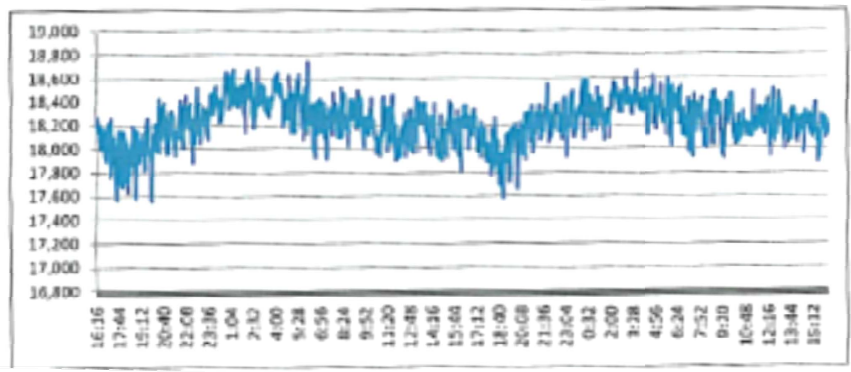

Figure 13: voltage pattern of the switch reactor for one operating day

Based on the filed data, when both reactors are out the voltage at the connection point of the switch reactor is about $17.9 \mathrm{kV}$. Similarly, in the case of one reactor in, the voltage level is about $18.02 \mathrm{kV}$ and finally when both reactors are in, the voltage at the connection pint of the switch reactor is about $18.4 \mathrm{kV}$.

The field data collected from the installation point of the switch reactor is completely in line with the simulation results and shows the smooth working of the installed switch reactor.

Next section would close this paper with its concluding remarks.

\section{CONCLUSION}

This paper deals with the design and study of a prototype switch reactor for central Queensland SWER system. The main issue of SWER systems is the charging current of parallel capacitors and its associated over voltage along with the occupied capacity of the transmission lines. The many fixed reactors installed in the central Queensland SWER system could mange the voltage rise and keeping the voltage in the permitted voltage band. However, the problem rises when the SWER system is heavy loaded and the fixed reactors make the situation much worse in terms of the provided voltage to the end-user consumers. Addressing the problem, this paper discussed the design and study of a contactor-based switch reactor which can adjust the connected reactors to the SWER system based on the sensed voltage level. For economic purposes, the design of the reactors has been done based on the $240 \mathrm{~V}$ which could be provided by the secondary of a SWER transformer.

The next step of this project would be optial allocation application of the designed switch reactor to the Central Queensland SWER system.

\section{REFRENCES}

[1] Process Corp., Framingham, MA. Intranets: Internet technologies deployed behind the firewall for corporate productivity. Presented at INET96 Annu. Meeting. [Online]. Available: http://home.process.com/ Intranets/wp2.htp

[2] L. Mandeno, "Rural power supply especially in back country areas," in Proc. Of New Zealand Institute of Engineers, vol. 33, 1947, ferguson and Osborn printers, Wellington, pp. 234-271

[3] N. Chapman, "Australia's rural customers benefit from single wire earth return systems," Trans. Dist., pp. 56-61, April 2001.

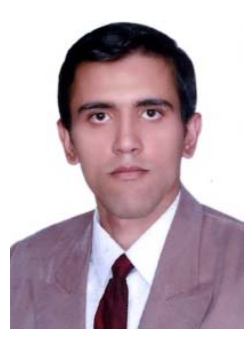

Mohammad R. Hesamzadeh (GSM'08) is with CQ University, Australia, as a Senior Researcher. His special fields of interest include high voltage transmission system design and planning, distribution and rural system studies, electricity market analysis, intelligent system applications in power systems and high voltage engineering. He is a Graduate Student Member of IEEE, Vice Chair of IEEE, Queensland Power Engineering Society, and a Professional Engineer in Australia.

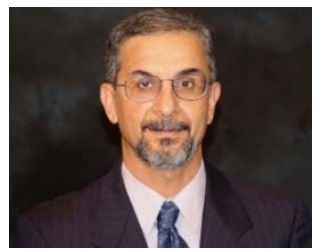

Nasser Hosseinzadeh (M'86) is currently the Head of Department of Systems at the Faculty of Sciences, Engineering and Health at Central Queensland University, Australia. He graduated from Shiraz University, Iran, in 1986 with a B.Sc. degree in electrical and electronics engineering. He worked in a research centre for five years before starting his postgraduate studies. He received a M.Sc. degree from Iran University of Science and Technology in 1992 and a Ph.D. degree from Victoria University in Australia in 1998. His special fields of interest include power system analysis and planning, power system stability, intelligent system applications in engineering, distribution networks and rural energy supply.

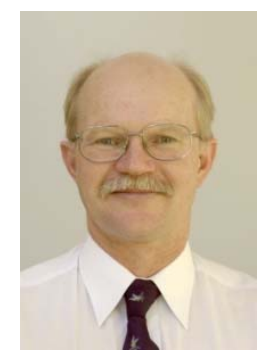

Peter J. Wolfs (M'80-SM'99) was born in Rockhampton Australia in 1959. He graduated from the Capricornia Institute of Advanced Education in 1980 with a B.Eng. degree. He subsequently obtained the M.Eng. degree from the Philips International Institute in the Netherlands in 1981 and the Ph.D. degree at the University of Queensland in 1992. He is the Associate Dean (Research and Innovation) at the Faculty of Sciences, Engineering and Health at Central Queensland University. His special fields of interest include rural and renewable energy supply, solar and hybrid electric vehicles and intelligent systems applications in railways. Professor Wolfs is a Fellow of Engineers Australia, a Registered Professional Engineer in the State of Queensland and a member of the Railway Technical Society of Australia. 